\title{
PERFIL VOCAL DO GUIA DE TURISMO
}

\author{
Vocal profile of tourism guide
}

Elisângela Barros Soares ${ }^{(1)}$, Carla Maria Cavalcanti Padilha de Brito (2)

\begin{abstract}
RESUMO
Objetivo: caracterizar o perfil vocal dos guias de turismo, bem como gênero e idade. Métodos: participaram desse estudo 23 guias de turismo, de ambos os gêneros, com idade entre 25 a 64 anos, participantes do Sindicato de Guias de Turismo do Estado de Pernambuco, que compareceram às reuniões trimestrais no período da coleta. Trata-se de um estudo de caráter descritivo, observacional e transversal. Para coleta foi realizada avaliação perceptivo-auditiva GRBAS. Resultados: observouse que a maioria dos guias apresentou loudness adequada, pitch normal e voz alterada. Além disso, as médias dos tempos máximos de fonação das vogais e das fricativas encontravam-se reduzidas e ataque vocal isocrônico. A ressonância, na maioria dos guias, estava equilibrada, mas houve uma incidência de ressonância laringo-faringea. A articulação foi precisa, com tipo e modo respiratório misto e nasal, respectivamente. Quanto à escala GRBAS as alterações apareceram de forma leve no G (grau de alteração vocal) em $68 \%$. Conclusão: na amostra estudada, a maioria era do gênero feminino com média de idade de 46 anos, e perfil vocal caracterizado por tempo máximo de fonação reduzidos, relação s/z adequado, ataque vocal isocrônico, pitch normal, loudness adequado, qualidade vocal alterada, com presença de rouquidão, soprosidade, tensão. A ressonância da maioria estava equilibrada e a articulação precisa, com tipo e modo respiratório misto e nasal, respectivamente. Quanto à escala GRBAS, as alterações apareceram de forma leve no grau de alteração vocal (G) em $68 \%$ e tensão (S) em $78 \%$ dos sujeitos.
\end{abstract}

DESCRITORES: Fonoaudiologia; Perfil de Saúde; Voz; Turismo

\section{INTRODUÇÃO}

A voz exerce um papel fundamental na comunicação e no relacionamento humano, enriquecendo a transmissão da mensagem articulada, acrescentando à palavra, o conteúdo emocional, a entoação, a expressividade, identificando o indivíduo tanto quanto sua fisionomia e suas impressões digitais ${ }^{1}$.

Nos dias de hoje, existem profissões que exigem que o indivíduo utilize a sua voz de forma contínua, como por exemplo, professor, cantor, locutor, ator, pastor. Aestes indivíduos que usam a voz no desempenho profissional, denominam-se profissionais da voz.

Muitos são os estudos direcionados aos profissionais da voz ${ }^{2-11}$. Em um levantamento das publicações brasileiras, sobre a voz do professor foi verificado que no período de 1993 a 1999 mais de 55 trabaIhos foram publicados ${ }^{12}$. Esse número com certeza já cresceu muito e o mesmo acontece com os demais profissionais que utilizam a voz como instru-

Fonoaudióloga do Memorial Hospital de Goiana - PE.

2) Fonoaudióloga; Professora substituta do Curso de Fonoaudiologia da Universidade Federal de Pernambuco. mento de trabalho, contudo, o profissional guia de turismo, mesmo inserido na categoria de profissional da voz, é um grupo ainda pouco estudado na clínica fonoaudiológica.

Os problemas vocais, em profissionais da voz, têm um significado diferenciado daqueles que não precisam da voz para seu sustento ${ }^{13}$. Dessa forma, conhecer a voz desses profissionais, identificando também os parâmetros mais alterados, pode facilitar o desenvolvimento de ações mais direcionadas a minimizar problemas e maximizar as potencialidades.

O som produzido pelas pregas vocais é modificado pela faringe, palato mole, língua e pelos lábios, produzindo sons individuais da fala ${ }^{14}$. O conjunto de características de determinada voz é denominado qualidade vocal, a qual pode ser avaliada por meio de uma avaliação perceptivo-auditiva ${ }^{15}$.

O julgamento da qualidade vocal é uma das maiores dificuldades da análise perceptivo-auditiva, pois envolve desde aspectos sócio-econômicos e culturais até preferências individuais ${ }^{16}$.

A qualidade vocal refere-se ao processo de identificar o tipo de voz utilizado pelo indivíduo e está relacionado com a seleção de ajustes motores emprega- 
dos, tanto em nível de pregas vocais e laringe, quanto em nível do sistema de ressonância. Além dos dados relativos às escolhas anatômicas o tipo de voz carrega elementos de outras duas dimensões, psicológica e sócio-educacional ${ }^{15,17}$.

Existem muitas formas de classificar os tipos de voz, levando-se em consideração a diversidade de aspectos que podem ser contemplados. A terminologia utilizada depende do estudioso que a define e várias classificações surgiram ao longo do tempo ${ }^{14,15,18,19}$.

A avaliação perceptivo-auditiva da voz, apesar de ser considerada subjetiva, é um referencial na terapia fonoaudiológica, pois averigua o progresso do tratamento instituído, facilita a compreensão da fisiologia dos órgãos da voz, direciona a terapia fonoaudiológica, sensibiliza o indivíduo quanto as suas necessidades de mudança de comportamento e auxilia no esclarecimento de fatores causais da desordem ${ }^{14,15,18,20}$.

No processo de avaliação vocal, parâmetros são analisados no intuito de dimensionar o comportamento vocal produzido e a seleção de parâmetros vai depender do objetivo da avaliação ${ }^{14-16,18}$.

Esses parâmetros são formados por: tipo de voz, sistema de ressonância, freqüência, intensidade, medidas fonatórias, coordenação pneumofonoarticulatória, entre outros ${ }^{15}$.

A avaliação perceptivo-auditiva precede a conclusão da existência ou não de uma disfonia e sua confiabilidade depende da experiência prévia do analisador, o que reduz o grau de subjetividade ${ }^{21}$.

Estudo mostrou que existe concordância entre a avaliação fonoaudiológica perceptivo-auditiva e as avaliações médicas, bem como os exames médicos entre si no diagnóstico de alterações vocais e/ ou laríngeas ${ }^{22}$.

Graças à cooperação dos otorrinolaringologistas, fonoaudiólogos e professores da voz, aumentaram a habilidade dos clínicos em avaliar e tratar patologias vocais, nos profissionais da $\mathrm{VOz}^{23}$.

Outra forma de avaliar a voz pode ser realizada por meio de uma escala desenvolvida pelo Comitê Phonatory Function Tests da Japan Society of Logopedics uns Phoniatrics, denominada pelos hispânicos de escala GRBAS. Escala japonesa, usada internacionalmente, que avalia o grau global da disfonia (G), e outros fatores como a rugosidade, a soprosidade, a astenia e a tensão, usando-se pontos para identificar o grau do desvio, onde "0" (zero) - normal ou ausente, "1" - discreto, "2" -moderado, "3" - severo ${ }^{15}$.

Como não foi encontrado nesta pesquisa estudo voltado para a avaliação da voz do guia de turismo, e devido à importância do tema, o objetivo desta pesquisa é obter o perfil vocal desses profissionais, por meio de uma avaliação perceptivo-auditiva baseada nos parâmetros propostos na literatura ${ }^{15} \mathrm{e}$ escala GRBAS, além de caracterizar o gênero e a idade desses profissionais.

\section{MÉTODOS}

A presente pesquisa foi desenvolvida no Sindicato dos Guias de Turismo de Pernambuco (SINGTURPE), localizado na Casa da Cultura na cidade do Recife, com 23 guias de turismo sindicalizados.

Os indivíduos foram selecionados obedecendo-se os seguintes critérios de inclusão: presença na reunião trimestral do sindicato no período de coleta, ter o curso de guia de turismo, sendo cadastrado na EMBRATUR (Empresa Brasileira de Turismo) como guia regional, nacional, internacional, especializado em atrativos naturais ou especializado em atrativos culturais e fazer parte do sindicato dos guias de turismo de Pernambuco (SINGTUR), independente de gênero, idade e tempo de profissão.

Os critérios de exclusão foram: ausência na reunião trimestral do sindicato, não ter o curso de guia de turismo e não ser cadastrado na EMBRATUR.

A coleta foi realizada entre os meses de setembro 2004 a março de 2005, em duas reuniões trimestrais. Trata-se de uma pesquisa observacional, transversal, descritiva, do tipo série de casos. Foi realizada a gravação da voz por meio de um gravador Aiwa TP-VS 535 com fita TDK 60 mim e os guias de turismo sindicalizados, assinaram o termo de consentimento. Neste momento, foi solicitado, pela pesquisadora, que o profissional emitisse as vogais /a/, /i/, $/ \mathrm{u}$, as fricativas /s/ e /z/ para medir o tempo máximo de fonação, após uma inspiração, e a contagem de números de 1 a 20 , sendo os tempos medidos por meio de um cronômetro (Technos Skydiver Profesional).

Foi solicitado a cada indivíduo que emitisse a vogal /a/ pelo maior tempo possível após inspiração. $O$ mesmo foi realizado na emissão das vogais /i/ e /u/, das fricativas $/ \mathrm{s} / \mathrm{e} / \mathrm{z} /$. Também foi solicitada a contagem de números de modo natural.

O grau global da disfonia (G) foi avaliado a partir da identificação de quatro fatores: rugosidade $(R)$ que engloba o conceito de rouquidão, crepitação, bitonalidade e aspereza; soprosidade (B); astenia (A) e tensão (S), sendo os fatores astenia e tensão excludentes entre si. Usaram-se pontos para identificar o grau do desvio, onde "O" (zero) - normal ou ausente, "1" - discreto, "2" -moderado, "3" - severo ".

A análise perceptivo-auditiva foi baseada em trabalho da literatura ${ }^{15}$, avaliando-se os seguintes parâmetros:

Pitch - referente à sensação subjetiva de freqüência fundamental. Foi classificado como normal agudo ou grave.

Loudness - julgamento do som em forte, fraco ou adequado.

Tipos de voz - padrão básico de emissão vocal de um indivíduo. Foi classificado como: voz rouca, áspera, soprosa, sussurrada, tensa, trêmula, 
infantilizada, feminilizada, virilizada, crepitante, presbifônica, hipernasal e adaptada.

Sistema de ressonância: conjunto de elementos do aparelho fonador que se relacionam entre si, visa à moldagem e a projeção do som no espaço. Foi classificado como ressonãncia equilibrada; laringofaríngea, faríngea, oral ou nasal, a partir dos seguintes critérios:

- ressonância equilibrada: quando apresenta riqueza de harmônicos amplificados na voz, e liberdade muscular de modificar esses ajustes.

- ressonância laringo-faríngea: quando há tensionamento conjunto dessas duas regiões e a voz apresenta-se com qualidade vocal comprimida ou tenso-estrangulada

- ressonância faríngea: quando há uso excessivo da faringe, conferindo uma emissão geralmente tensa, com característica metálica na voz.

- ressonância oral: quando há concentração de energia na cavidade da boca, com uma articulação dos sons da fala demasiadamente trabalhada (sobrearticulação).

- ressonância nasal: quando observa-se o uso excessivo ou uso insuficiente da cavidade nasal, comumente associada às afecções que envolvem alterações da anatomofisiologia do palato mole, tais como fissura palatina, insuficiência ou incompetência velofaríngea.

Tipo articulatório: refere-se ao ajuste dos órgãos fonoarticulatórios na produção e formação do som, pode ser classificada em precisa, indiferenciada, exagerada ou travada A articulação precisa geralmente ocorre nos profissionais da voz e transmite ao ouvinte franqueza, desejo de ser compreendido e clareza de idéias ou indicativo de normalidade. Aarticulação mal definida pode indicar dificuldades na organização mental, não preocupação em ser compreendido ou mesmo falta de vontade de se comunicar. Por sua vez, uma articulação exagerada indica certo grau de narcisismo.

Dinâmica respiratória: considerando os quatro padrões de tipo respiratório: superior (elevação da clavícula na inspiração e pouca movimentação do abdômen), inferior (não ocorre movimentação da região superior do tórax sendo o abdômen o responsável pela maior parte do movimento), misto (encontrando os dois padrões associados) ou diafragmáticaabdominal ou costodiafragmática (caracterizada pela expansão harmônica da caixa torácica, sem excessos na região superior ou inferior, sendo a mais eficaz para o desenvolvimento da voz profissional). $O$ modo: nasal (predominantemente pelo nariz), bucal (predominantemente pela cavidade oral) e oro-nasal (utilizando os dois modos de forma alternada); e a coordenação pneumofonoarticulatória (C.P.F.A.), referindo-se ao resultado da inter-relação harmônica das forças expiratórias, mioelásticas da laringe e musculares da articulação. Podendo ser considerada adequada (bom controle no uso do ar) e inadequada (utilização do ar de reserva, uso de muito ar no início da fonação ou quando as inspirações são constantes e/ou ruidosas).

A análise da voz foi realizada pelas pesquisadoras, uma delas especialista em voz, baseadas no consenso de julgamento de ambas, a partir da gravação da contagem de números, da emissão das vogais /a/, /i/, /u/ e fricativas /s/ e /z/, que permitiram uma investigação quantitativa e qualitativa da fonação dos sujeitos.

Por meio da emissão das vogais foi possível verificar a ressonância, a qualidade vocal, além de perceber a habilidade do indivíduo em controlar as forças aerodinâmicas da corrente pulmonar e as forças mioelástica da laringe. Na sustentação das fricativas médias surdas e sonoras /s/, /z/ verificou-se se o indivíduo utilizava a suplência de ar pulmonar de modo eficiente. Na fricativa/s/ observou-se o suporte aéreo pulmonar e a habilidade de controlá-lo, e na /z/ o comportamento vocal resultante.

A contagem de números, reproduzida de modo mais aproximado com a emissão vocal da fala, foi realizada após uma inspiração profunda, em altura, intensidade, qualidade e velocidade de fala habitual, podendo-se avaliar alguns parâmetros como tipo de voz, pitch, loudness, articulação, entre outros. Essa contagem forneceu dados da eficiência do individuo para controlar a respiração e a fonação no processo de fala encadeada. Com o tempo máximo de fonação (TMF) verificou-se também se o indivíduo apresentava ataque vocal, e a estabilidade da fonação na qual esta requer um acurado controle do sistema nervoso central.

O estudo foi aprovado pelo Comitê de Ética em Pesquisa da Universidade Federal de Pernambuco, sob número de processo 215/2004.

Os dados foram apresentados na forma de estatística descritiva utilizando-se o programa Excel, com freqüências absolutas e relativas, bem como medidas de tendência central.

\section{RESULTADOS}

A população estudada é composta em sua maioria pelo gênero feminino $(69,57 \%)$, com média de idade de 46 anos (Tabela 1).

A média do tempo máximo de fonação das vogais /a/, /i/, /u/ foi de oito e nove segundos e média das fricativas /s / e /z/ encontram-se reduzidas, verificando-se que há um grande intervalo entre a menor e a maior emissão de fricativas, contudo a média da relação s/z encontra-se adequada (Tabela 2).

Com relação ao ataque vocal apresentaram $65,2 \%$ isocrônico e $34,8 \%$, brusco. Concernente ao pitch apresentado, $74 \%$ foi normal e $26 \%$, grave. A loudness mostrou-se adequada em $65 \%$ dos guias de turismo, fraca em $22 \%$ e forte em $13 \%$. 
Referente à qualidade vocal dos guias de turismo, $43 \%$ possuíam a voz adaptada; $4,35 \%$ voz rouca; $4,35 \%$ voz soprosa; $13 \%$ voz presbifônica; $4,35 \%$ voz áspera; $8,7 \%$ crepitante e por fim, $21,75 \%$, outros (4,35\%, voz adaptada com tensão; $4,35 \%$ rouca e trêmula; $4,35 \%$ rouca e tensa; $4,35 \%$ soprosa e trêmula e $4,35 \%$ rouca e soprosa) (Tabela 3 ).

Quanto à ressonância, $48 \%$ apresentaram equilíbrio na ressonância, 39\% ressonância laringo- faríngea; $9 \%$ faríngea e $4 \%$ nasal (Tabela 4).

Quanto à articulação, todos obtiveram precisão na articulação. Com relação ao tipo respiratório, $83 \%$ foram do tipo misto, $13 \%$ superior e $4 \%$ inferior ou abdominal.

Quanto ao modo respiratório em repouso 43,5\% apresentaram padrão oro-nasal e 56,5\% nasal.

$\mathrm{Na}$ escala de GRBAS, observa-se que $68 \%$ apresentaram grau global da disfonia leve (Tabela 5).

Tabela 1 - Distribuição dos guias sindicalizados no SINGTUR-PE quanto à idade

\begin{tabular}{lcccc}
\hline & Mínimo & Máximo & Média & Moda \\
Idade (anos) & 25 & 64 & 46,8 & 44 \\
\hline
\end{tabular}

SINGTUR-PE - Sindicato de Guias de Turismo de Pernambuco

Tabela 2 - Distribuição do tempo máximo de fonação e a relação s/z

\begin{tabular}{l|c|c|c}
\hline & Mínimo & Máximo & Média \\
la/ & 2,8 & 13 & 8,3 \\
li/ & 2,8 & 23 & 9,1 \\
/u/ & 2 & 23 & 9,3 \\
/s/ & 3 & 16 & 7,9 \\
lz/ & 2,3 & 14 & 7,6 \\
$\mathrm{~s} / \mathrm{z}$ & 0,7 & 1,7 & 1,1 \\
\hline
\end{tabular}


Tabela 3 - Distribuição em percentual da qualidade vocal

\begin{tabular}{c|c} 
Rouca & $4,35 \%$ \\
Soprosa & $4,35 \%$ \\
Presbifônica & $13 \%$ \\
Áspera & $4,35 \%$ \\
Crepitante & $8,70 \%$ \\
Adaptada & $43,50 \%$ \\
Outros & $21,75 \%$ \\
\hline
\end{tabular}

Tabela 4 - Distribuição em percentual da ressonância

\begin{tabular}{c|c}
\hline Equilibrada & $48 \%$ \\
Laríngo-faríngea & $39 \%$ \\
Faríngea & $9 \%$ \\
Nasal & $4 \%$ \\
\hline
\end{tabular}

Tabela 5 - Distribuição em percentual da quanto a escala GRBAS

\begin{tabular}{cc|c|c|c}
\hline & "0"- Normal & "1"- Leve & "2" - Moderado & "3" - severo \\
G & $32 \%$ & $68 \%$ & - & - \\
R & $78 \%$ & $22 \%$ & - & - \\
B & $44 \%$ & $56 \%$ & - & - \\
A & $87 \%$ & $13 \%$ & - & - \\
S & $22 \%$ & $78 \%$ & - & - \\
\hline
\end{tabular}




\section{DISCUSSÃO}

Verifica-se que o tempo máximo de fonação das vogais esperado para mulheres é de 14 a 25 segundos e para homens, de 20 a 35 segundos, sendo que o presente estudo mostrou que na emissão das vogais, este parâmetro está aquém do normal. Observou-se que a média das fricativas $/ \mathrm{s} / \mathrm{e} / \mathrm{z} /$ encontravam-se reduzidas, verificando-se um grande intervalo entre a menor e a maior emissão. A média desejável sugerida para esses valores é entre 15 a 25 segundos, sendo abaixo de 15 segundos indicativo de comprometimento de suporte aéreo ${ }^{21}$.

Uma relação s/z maior ou igual a 1/2 é indicativa de falta de coaptação correta das pregas vocais na fonação ${ }^{16,20}$, porém na amostra estudada esta relação encontrava-se adequada.

$\mathrm{O}$ ataque vocal isocrônico é o adequado, já que neste caso a fase expiratória coincide com o início da vibração da mucosa das pregas vocais ${ }^{14} \mathrm{e}$ esse foi o parâmetro obtido na maioria das avaliações dos guias, contudo o ataque brusco também esteve presente. $O$ ataque vocal brusco é definido como um dos exemplos de mau uso, pois ocorre uma rápida e completa adução das pregas vocais antes da iniciação da fonação ${ }^{14}$. Este estado pode ser acompanhado de tensão muscular e quando presente em grau significativo no falante, dá evidências de esforço aumentado ${ }^{14}$.

O pitch apresentou-se, em sua maioria, normal, mas houve um percentual alterado, do tipo grave.

O percentual da loudness foi favorável, apesar de ser importante também essa avaliação in loco, pois foi referido pelos guias a utilização da intensidade excessiva geralmente usada para manter a atenção dos ouvintes, e isso pode levar a mudanças teciduais, resultando em lesões locais orgânicas no ponto de maior força de contato das pregas vocais. A intensidade deve ser adequada ao ambiente em que estamos falando, a fim de evitar danos à voz, visto que uma intensidade elevada pode ser considerada como um dos fatores de risco à voz ${ }^{24}$. O profissional guia de turismo possui exageros na intensidade ${ }^{25}$, contudo nesse estudo a loudness foi adequada em um número significativo de guias.

A qualidade vocal é o principal parâmetro da análise perceptivo-auditiva, fornecendo informações sobre o padrão básico da emissão e sobre as dimensões biológica, psicológica e sócio-educacionais da fala $^{21}$. Na avaliação perceptivo-auditiva, a qualidade vocal estava alterada na maioria dos profissionais, sendo observado vozes roucas, soprosas, trêmulas, tensas, presbifônicas, ásperas e crepitantes. Esses tipos de qualidade vocal encontradas no estudo indicam prováveis lesões nas pregas vocais, justificando com isso, esse parâmetro vocal ou pode representar uma predisposição a alterações glóticas e vocais que, com o passar do tempo, podem desenvolver-se ${ }^{26}$.

É importante que haja o uso consciente da voz, por parte desses guias de turismo, a fim de garantir a saúde dos profissionais e reduzir riscos de lesões nas pregas vocais ${ }^{27}$. Os serviços de fonoaudiologia também devem ser divulgados, para que eles saibam a quem recorrer e possam receber as orientações necessárias, pois grande parte dos distúrbios vocais pode ser evitada pela mudança de alguns hábitos ${ }^{28,29}$.

As alterações da qualidade vocal ocorrem, na maioria das vezes, de forma lenta e gradual e o indivíduo pode não perceber as modificações da voz devido à perda da referência do padrão vocal saudável que possuía anteriormente ${ }^{10}$.

Verifica-se que a maioria possui ressonância equilibrada, com liberdade muscular de modificar esses ajustes. No entanto, 39\% têm uma ressonância "presa na garganta", caracterizando o uso excessivo da laringe com tensão e projeção inadequada no ambiente ${ }^{15}$.

De forma geral, a presença dos sintomas de rouquidão, alcance de pitch reduzido, fadiga vocal e sensação de pressão na garganta causam impacto negativo no desempenho, metas de carreira, saúde física e psicológica, dos profissionais atores, como também em cantores, professores e mulheres instrutoras do exército ${ }^{30}$. Esses sintomas apareceram na amostra de guias estudada, trazendo provavelmente o mesmo tipo de impacto negativo na performance desses profissionais.

A articulação precisa encontrada nesses profissionais indica controle na dinâmica fonoarticulatória. A literatura refere que esse padrão estaria associado à transmissão de franqueza e clareza de idéias ao ouvinte, além de ser a articulação esperada em um profissional da voz ${ }^{15}$.

Quanto à respiração a maioria apresentou o tipo misto, que é o mais comumente observado na população, apresentando pouca movimentação superior e inferior durante a inspiração, utilizado em repouso, contudo é inadequada na atuação profissional ${ }^{15}$. Referente ao modo respiratório, em repouso, o esperado é que a inspiração seja nasal, sendo assim, a maioria esta adequada, aspecto este mais observado na presente pesquisa.

$\mathrm{Na}$ escala de GRBAS, observa-se que $68 \%$ apresentaram grau global da disfonia discreto e que a presença de tensão (S) com 78\%, concorda com a tensão observada na qualidade vocal.

\section{CONCLUSÃO}

Os guias de turismo sindicalizados no SINGTUR$P E$ são em sua maioria do gênero feminino com média de idade de 46 anos. O perfil vocal desse profissional foi caracterizado por um TMF das vogais e fricativas reduzidos, relação $\mathrm{s} / \mathrm{z}$ adequado, ataque vocal 
isocrônico, pitch normal, loudness adequado, qualidade vocal alterada, com presença de rouquidão, soprosidade, tensão. A ressonância da maioria foi equilibrada, contudo houve alta incidência de ressonância laringo-faríngea. A articulação foi precisa, com tipo e modo respiratório misto e nasal, respectivamente.

Quanto à escala GRBAS, as alterações apareceram na maioria da amostra de forma leve no grau de alteração vocal (G) e tensão (S).
Desta forma, verifica-se que apesar dos guias estudados não apresentarem grandes alterações nos parâmetros estudados é importante um trabalho de aperfeiçoamento vocal, por serem profissionais da voz, visando à prevenção de problemas vocais, assim como se deve realizar novas pesquisas com esses profissionais sendo avaliados in loco para identificar e eliminar os abusos e mau uso da voz, podendo garantir um desempenho vocal eficaz.

\section{ABSTRACT}

Purpose: to characterize the vocal profile of tourism guides, as well as gender and age. Methods: 23 guides took part in this study, of both genders, with age between 25 to 64 years, partakers of the Union of Tourism Guides of the State of Pernambuco, who appeared to the quarterly meetings in the period of the collection. It is a descriptive, observational and crossed-nature study. A perceptual-auditive analysis was carried out for the sampling Results: we noted that the majority of the guides demonstrated adequate loudness, normal pitch and modified voice. Moreover, the averages of the maximum times of phonation of the vowels and the affricative and isocronic vocal attack were reduced. The resonance, in the majority of the guides, was balanced, but it had an incidence of laryngeal - pharyngeal resonance. Articulation was precise, with mixed and nasal respiratory mode and type, respectively. As for GRBAS scale, the alterations appeared in light form in $\mathrm{G}$ (degree of vocal alteration) in $68 \%$. Conclusion: in the studied sample, the majority was of the feminine gender with average age of 46 years, and vocal profile characterized by reduced maximum phonation time, adequate $s / z$ relation, adequate isocronic vocal attack, normal pitch, loudness, modified vocal quality, with presence of roughness, breathiness, tension. The resonance of the majority was balanced and articulation precise, with mixed and nasal respiratory mode and type, respectively. As for GRBAS scale, the alterations appeared in light form in G (degree of vocal alteration) in $68 \%$ and $S$ (tension) in $78 \%$ of the subjects.

KEYWORDS: Speech, Language and Hearing Sciences; Health Profile; Voice; Tourism

\section{REFERÊNCIAS}

1. Pedroso MIL. Técnicas vocais para os profissionais da voz. [monografia]. São Paulo (SP): Centro de Especialização em Fonoaudiologia Clínica; 2000.

2. Campiotto AR. Atuação fonoaudiológica no trabaIho com cantores. In: Lopes Filho O, editor. Tratado de fonoaudiologia. São Paulo: Roca; 1997. p. 72333.

3. Chun RYC, Dijk EV, Furlan CE, Silvério KCA. Voz profissional: grupos de voz na comunidade de Piracicaba. In: Ferreira LPC, Costa HO. Voz ativa: falando sobre o profissional da voz. São Paulo: Roca; 2000. p. 80.

4. Dragone MLOS, Behlau M. Ocorrência de disfonia em professoras: fatores relacionados com a voz profissional. In: Behlau M. Voz do especialista. v. 1. Rio de Janeiro: Revinter; 2001. p. 28.

5. Fabron EMG, Omote S. Queixas vocais entre professores e outros profissionais In: Ferreira LPC, Costa HO. Voz ativa: falando sobre o profissional da voz. São Paulo: Roca; 2000. p. 91-100.
6. Bacha SMC, Camargo AFFP, Brasil MLR, Monrel VRFC, Nakao EMH, Rocha AE, Tutes ER, Nakao M. Incidência de disfonia em professores de pré-escola do ensino regular da rede particular de Campo Grande / MS / Brasil. Pró-Fono R Atual Cient. 1999; 11(2):8-14. 7. Navarro CA, Behlau M. Perfil vocal dos locutores: profissionais da voz em publicidade. In: Behlau M. Voz do especialista. v. 1, Rio de Janeiro: Revinter; 2001. p. 57-76.

8. Ortiz E, Costa EA, Spina AL, Crespo AN. Proposta de modelo de atendimento multidisciplinar para disfonias relacionadas ao trabalho: estudo preliminar. Rev Bras Otorrinolaringol. 2004; 70(5):20-3.

9. Santiago RF, Brito CMP. Hábitos de higiene vocal dos locutores de rádio da cidade do Recife [monografia]. Recife (PE): Graduação em Fonoaudiologia; Universidade Federal de Pernambuco; 2004.

10. Scalco MAG, Pimentel RM, Pilz WA. Saúde vocal do professor: levantamento junto a escolas particulares de Porto Alegre. Pró-Fono R Atual Cient.1996; 8(2):25-30.

11. Silva MAA. Voz profissional: novas perspectivas 
de atuaçäo. Rev Disturb Comun. 1999; 10(2):177-92. 12. Viola IC, Ferreira LP, Sene CD, Villas-Boas DC, Souza SM. A voz do professor: levantamentos das publicações brasileiras. Rev Soc Bras Fonoaudiol. 2000; 5(7):36-47.

13. Petroucic RT, Friedman S. Os sentidos da perda da voz. Rev Disturb Comun. 2006; 18(1):39-49.

14. Colton $\mathrm{RH}, \mathrm{Casper} \mathrm{JK}$. Compreendendo os problemas de voz. Porto Alegre: Artes Médicas; 1996.300 p. 15. Behlau M, Madazio G, Feijó D, Pontes P. Avaliação da voz. In: Behlau M, organizadora. Voz: o livro do especialista . v. 1. Rio de Janeiro: Revinter; 2001. p. 91-113. 16. Master S, Biase N, Pedrosa V, Chiari BM. O espectro médio de longo termo na pesquisa e na clínica fonoaudiológica. Pró-Fono R Atual Cient. 2006; 18(1):111-20.

17. Behlau MS, Ziemer R. Psicodinâmica vocal. In: Ferreira LP. Trabalhando a voz. São Paulo: Summus; 1988. p. 71-88.

18. Boone DR, McFarlane SC. A voz e a terapia vocal. 5. ed. Porto Alegre: Artes Médicas; 1994.350 p.

19. Fawcus M. As causas e classificações dos distúrbios de voz. In: Frledmam M, Fawcus M. Distúrbios da voz e seu tratamento. 3. ed. São Paulo: Santos; 2004. p. 56-8.

20. Fukuyama EE. Análise acústica da voz captada na faringe próximo à fonte glótica através de microfone acoplado ao fibrolaringoscópio. Rev Bras Otorrinolaringol. 2001;67(6):776-86.

21. Behlau M, Pontes P. Avaliação e tratamento das disfonias. São Paulo: Lovise; 1995. 290 p.

22. Nemr K, Amar A, Abrahäo M, Leite GCA, Köhle
JS, Santos AO, Correa LAC. Análise comparativa entre avaliação fonoaudiológica perceptivo-auditiva, análise acústica e laringoscopias indiretas para avaliação vocal em população com queixa vocal. Rev Bras Otorrinolaringol. 2005; 71(1):13-7.

23. Sataloff RT, Spiegel JR. Care of the professional voice. Otolaryngol Clin North Am. 1991; 24(5):1093124.

24. Behlau M, Dragone MLS, Nagano L. Voz que ensina: o professor e a comunicação oral em sala de aula. Rio de Janeiro: Revinter; 2004. 60 p.

25.Behlau M, Feijó D, Madazio G, Rehder MI, Azevedo R, Ferreira AE. Voz profissional: aspectos gerais e atuação fonoaudiológica. In: Behlau M, organizadora. Voz: o livro do especialista. v. 2. Rio de Janeiro: Revinter; 2005. p. 298-9.

26. Corazza VR, Silva VFC, Queija DS, Dedivitis RA, Barros APB. Correlação entre os achados estroboscópicos, perceptivo-auditivos e acústicos em adultos sem queixa vocal. Rev Bras Otorrinolaringol. 2004; 70(1):30-4.

27. Simões M. O profissional de educaçäo física e o uso da voz: uma contribuiçäo da fonoaudiologia. Rev Bras Ativ Fís Saúde. 2000; 5(1):71-80.

28. Zeine $L$, Waltar KL. The voice and its care survey finding from actors perspectives. J Voice. 2002; 16(2):229-43.

29. Carelli EG, Nakao M. Educação vocal na formação do docente. Rev Fono Atual. 2002; 5(22):40-52. 30. Sapir S, Keidar A, Mathers-Schmidt B. Vocal attrition in teachers: survey findings. Eur $\mathrm{J}$ Disord Commun. 1993; 28(2):117-85.

Recebido em: 06/06/2006

Aceito em: 10/10/2006

Endereço para correspondência:

Rua Manoel Nascimento Torres, quadra-k, Lote 1, no 30

Goiana - PE

CEP: $55900-000$

Tel: (81) 36260713 / 88174744

E-mail:elissoares@hotmail.com ; elissoares2000@yahoo.com.br. 